very small limits, occur at the same places during delivery and in the same manner as when rigid forceps are used, traction being applied by the handles. Thus extension, for example, could take place without advance of head and forceps only by the forceps leaving the pelvic curve.

$A$ point which is not much dwelt on by inventors is that forceps which are best suited for axis traction, having a full pelvic curve, are unsuited for rotation and that if they rotate they are no longer axis-traction forceps. Indeed, the only way to restore true axis traction in such cases would be to rake them off after rotation and obtain a new grip. Forceps with a pronounced pelvic curve are evidently intended to be put on in the transverse diameter, and if rotated to any extent the pelvic curve is no longer a guide or indicator but rather a hindrance. Hence the wisdom shown by Simpson in making his forceps with a modified pelvic curve to allow of some rotation and slight movement of flexion or extension. It seems to me a problem worthy of grave attention whether for general use the axis-traction forceps coming so much into vogue are not more dangerous than Simpson's long forceps used with some knowledge and caution. Rigby in his "System of Midwifery" says: "The forceps is the simplest imitation of nature, for, in fact, it is notbing more than a pair of artificial hands introduced one on each side of the head." Now when a man grasps the handles of the forceps the instrument may be regarded as a continuation of his own hands. There is direct connexion between his hands and the footus, he feels when it moves or yields, estimates more accurately the force he is using, and by his great leverage can, guided by his knowledge, help its progress at a difficult point. Using the axistraction forceps is like putting another man's hands into the pelvis to grasp the head and then pulling on his coat-tails. I am so convinced $o$ the advantage of a rigid connexion with the fœetus that, apart from other considerations, I consider that to be one of the advantages of Murdoch Cameron's antero-posterior forceps. It is quite common for medical men to complete delivery by pulling on the traction bar. Now I confess that if the head is high in the pelvis, and indeed until it has reached the floor, it is of advantage to have an indicator of the direction of the axis and also the power of pulling freely in the direction of the axis, but $I$ am of opinion that in every case both hands should be used, one grasping the handles of the forceps and the other the traction handle. The hand which grasps the handles of the forceps can apply leverage where necessary, steady the progress of the foetus, and obtain information of that progress which would not be obtained by merely pulling on the traction handle. When the head has reached the floor of the pelvis it is especially necessary, for the sake of the perineum, among other reasons, to complete delivery without having recourse to the traction handle. In his "System of Midwifery," 1844, Rigby writes: "In extracting the head we must bear in mind the part of the pelvis in which it is impacted and make our efforts in the direction of its axis ; we must also recollect the curved form of the instrument, and that we must not pull in the direction in which the handles point, but rather hold them firmly in one hand and by pressing against the middle of the forceps with the othor guide the head downwards and backwards into the carity of the pelvis. We shall thus make our extractive effort in the direction of the upper portion of the blades. or that part which has the chief hold upon the head." (The italics are mine.) Here there is perfect axis traction arranged for without any great apparatus but simply by means of the ordinary forceps used with the good sense and ingenuity of a practical man, and his axis traction would be much more perfect than that of a practitioner who delivered by simply pulling on the traction handle of one of the latest instruments. Traction rods enable the operator to use the second hand to great advantage, but the more important band is the one which grasps the handles of the forceps. The forceps in Rigby's hands had another important use, for he continues: "Whilst extracting we should, as with the straight forceps, slowly move the handles from side to side and even make them describe a circle. We thus not only use the forceps as a simple extracting instrument, but make it act as a lever in every direction and greatly facilitate the advance of the head, even under circumstances of considerable impaction."

It was the considerations I have set forth which caused my forceps to be stillborn. I was providing for free movements of rotation, flexion, or extension, by placing immediately below each fenestra a swivel and a hinge, which could both be unlocked from the handles. I am now convinced. that while it is a benefit to be able to use the curved tractiom rods, the rigidity of the instrument and its simplicity shonle be interfered with as little as possible.

Glasgow.

\section{PARALYSIS AGITANS;}

WITH AN ACCOUNT OF A NEW SXMPTOM.

BY PURVES STEWART, M.A., M.D. EDIN., M.R.C.P. LOND.,

SENIOR HOUSE PHIYSTCIAN TO THE NATIONAI HOSPITAL FTOR THE PARALYSED AND EPILEPTIC, QUEEN-SQUARE, BLOOMSBURY, LONDON, W.C.

EIGHTx years ago Parkinson wrote his classical description of paralysis agitans. So little has been added to its symptomatology since and the disease now appears at first sight to be, clinically at least, so thoroughly worked out that to many it may seem a superfluous task to traverse once more a subject so well worn. The following paper, therefore, must be regarded as merely supplementary to the descriptions in the text-books, not in any sense as a substitute for them.

Within the past two years I have had opportunities of studying a number of patients, 28 in all, suffering from paralysis agitans and it is upon personal observations on these cases that the following remarks are based. Although the number of cases is not large enough to permit of any statistical references being founded upon them alone, yet the fact that they have been examined and compared by one person renders the value of the observations fairly uniform, whilst the cases are sufficiently numerous to justify me in drawing certain clinical conclusions. Of these 28 cases, 17 occurred in men and 11 in women. This greater frequency among male patients is in entire agreement with the combined statistics of a large number of cases seen by other physicians, which show the disease to be much more frequent in the male sex than in the female, the proportion being about 2 men to 1 woman.

The age of onset of symptoms in my series of cases is instructive. It ranges from twenty-two years to seventy-three years, the distribution being as follows:-In 1 case the earliest symptom appeared at the age of twenty-two years: in 1 case at thirty years; in 1 case at thirty-three years ; in 2 cases between the ages of thirty-five and forty years; in 3 cases between forty and forty-five years; in 6 cases: between forty-five and fifty years; in 7 cases between fifty and fifty-five years; in 4 cases between fifty-five and sixty years; in 2 cases between sixty and sixty-five years; and in 1 case at the age of seventy-three years. Thus 23 cases out of 28 -i.e., over 82 per cent.-commenced after the age of forty years.

A family history of nervous disease was obtained in only a few instances (in 6 cases out of 28). In 4 of these 6 cases there was epilepsy or insanity in the patient's brother or sister; in 3 of the 6 cases a relative had actually suffered from paralysis agitans-a maternal aunt in one case ${ }_{r}$ a paternal grandfather in another, and a sister in a third. As to exciting cause, 6 patients gave a history of fright or emotional disturbance. Thus, for example, in one case the earliest symptom-shaking of the left thumb-showed itself after a shock occasioned by a sister becoming insane. Another patient was at a menagerie when a lion escaped from its cage and caused considerable alarm amongst the spectators. On the next day the patient's illness is said to have commenced, his first symptom being stiffness of the left leg. Another patient was threatened as a "blackleg" by his fellow-workmen on the railway who were on strike and ever afterwards he found his right hand stiff and his handwriting unusually slow. Another patient blamed the shock of having a tooth extracted as being the antecedent cause of her illness, the earliest symptom in her case being tremor of the left hand. Worry or overwork was assigned as the exciting cause in 3 cases : 3 were said to have followed an attack of influenza; and one patient had an attack of right hemiplegia a few weeks before the tremor of paralysis agitans appeared in his left hand. One man had his foot trodden on by a horse a year before the onset of tremor in that foot. One woman's symptoms came on after a confinement at the age 
of forty-six years and another patient sustained a fall which is said to have produced a prolapse of the uterus and this was succeeded by stiffness of the legs. In the remaining 12 cases no cause was assigned by the patient in the form of injury, emotion, or preceding disease. Moreover, the severity of the disease in those cases where some exciting cause was ascribed was not at all proportional to the intensity of the assigned cause. It therefore seems probable, on the whole, that such so-called exciting causes, if they really are causes at all, merely precipitate the disease in a decaying nervous system.

The earliest symptom in a large proportion of my cases was not tremor but stiffness-contrary to the orthodox statements of the text-books. In 14 cases out of 28 stiffness and weakness preceded the tremor for periods varying from several weeks to over two years, and in 3 of these cases tremors were entirely absent although the disease had been well established, with its characteristic attitude and facial expression and with a definite history of stiffness and weakness, for fifteen months, eighteen months, and four years respectively. In 3 other cases, again, tremors only occurred on excitement or on voluntary effort, the patients exhibiting no tremor whatever when at rest. The dictum that the tremors of paralysis agitans are diminished by voluntary movement can therefore no longer be accepted as an invariable rule, nor as a secure point of diagnosis between paralysis agitans and other diseases.

The symptoms of the disease in most cases are hemiplegic in distribution. In only 2 cases out of 28 were the limbs of both sides equally affected. The left side appears in the majority of cases to be affected before the right, in righthanded people. Thus 14 cases began on the left side and only 12 on the right, and one of these 12 was in a lefthanded man, so that his case should be properly added to the 14 left-sided cases. That side which is first attacked remains, as a rule, more severely affected than the other side even when, later in the disease, the symptoms have become bilateral. The upper extremity was affected before the lower in 18 cases out of 28 and the hand usually suffered before the shoulder, though not invariably. Thus one patient, a woman, first felt her left hand weak and stiff when peeling potatoes, so that she had difficulty in turning the potato round. Another patient, a professional musician, first noticed weakness in her left hand when playing the organ. Several patients noticed, as their earliest symptom, an abnormal slowness in writing; another patient first experienced inconvenience from the tremor of his fingers owing to a difficulty in buttoning his clothes. In only one patient did the weakness begin in the shoulder-his attention being attracted by the unusual difficulty which he experienced in putting on his overcoat.

The orthodox clinical picture of a patient suffering from paralysis agitans is too well known to require much emphasising here. In a fully developed "classical" example of the disease the constant rhythmic tremors of fingers and wrists, the "interosseal" position of the fingers, the stooping attitude of the head and trunk, the "festinant" gait with its short, shuffling steps, the retropulsion on walking backwards, the difficulty in rising from the sitting to the standing posture, the tremors of the legs when sitting down, the slowness of voluntary movements in trunk and limbs, the fixed and sad facial expression,-all these, when present, form a striking clinical picture which it is easy to reeognise But such a condition of affairs is only reached when the malady is far advanced and we ought to be able to recognise its nature long before the disease has attained such severity as to be diagnosable telescopically.

Let us therefore consider some of the symptoms of the disease more in detail. The prevalent conception of paralysis agitans is that of a disease in which sensory symptoms are practically almost absent. Such, however, is not the case. Dull, aching pains in the affected limbs, along with a feeling of stiffness and weakness, often precede for a long time the actual rigidity or tremor. The patients also frequently complain of sudden flushes of heat or of cold spreading all over the body, especially at nights when in bed. As mentioned above, a case of paralysis agitans may run its course for years without any visible tremor whatsoever, this being the so-called "paralysis agitans sine agitatione." One reason why this variety or stage of the disease is not more generally known is because the real nature of the malady is usually not recognised until obvious tremors have appeared. As to motor symptoms, the presence of stiffness and rigidity should suggest paralysis agitans almost as readily as does the existence of visible rhythmic tremors. In paralysis agitans all voluntary movements may become slow and stiff, with a few notable exceptions, as in the ocular, palatal, and respiratory muscles. In a well-marked case, if the patient be requested to look suddenly at some object near the extreme lateral boundary of his field of vision, he turns his eyes smartly in the desired direction whilst his head and neck follow slowly and deliberately in the movement of rotation. Why the ocular muscles should usually escape it is difficult to explain, since almost all the other voluntary muscles of the face, trunk, and limbs may be affected, certainly by rigidity and often by tremor as well. Rhythmic tremor of the jaw occurred in 4 of my cases, the movement of the jaw being an up-and-down one and synchronous with the rhythmic movement of the limbs.

The face is characteristically affected, and in a well-marked case in which both sides of the face have become affected the result is the "Parkinsonian mask" in which the expression is fixed and woe-begone; it has been aptly termed the "starched" expression. In this condition the facial immobility is such that when the patient is talking his play of facial expression is almost nil, the deficiency being evident both in the upper and in the lower facial muscles. The condition can be most strikingly demonstrated by covering up the patient's chin and lower lip whilst he is talking. It is then often remarkable how motionless is the upper part of the face above and including the upper lip. And this "starched" expression of face may be unilateral, as will readily be understood when we remember the frequent hemiplegic distribution of the disease. Thus out of 11 cases which exhibited the phenomenon in no fewer than 9 the facial movements were distinctly asymmetrical, the weakness being most marked in the lower facial muscles and always on the same side as that on which the limbs were affected, the difference between the two sides of the face being best brought out when the patient was made to retract the upper lip, the naso-labial fold in such cases being distinctly shallower on the weaker side. In some cases ( 6 in my series) the face is not merely immobile but may actually develop rhythmic tremors, especially about the lips, and in 3 cases this was associated with a synchronous antero-posterior tremor of the tongue. The voice is often high-pitched and monotonous and its quality is frequently thin and quavering. The soft palate was not affected by tremors in any of my cases. Articulation is frequently disturbed, tending to assume a " festinant" character. The patient starts off deliberately enough at the beginning of a sentence, but after a few words the syllables become slurred and jumbled together, so that in some cases the concluding words of each sentence are almost unintelligible.

As regards the trunk and limbs the characteristic stooping attitude and the frequent rhythmic movements are so familiar that they need not be described here in detail. But one remark may be added-viz., that the attitude of the patient the "interosseal" position of the hand, the semi-flexed, pronated forearm, the frequently unilateral facial weakness, most evident in the lower facial muscles, the stiff, shuflling gait, often more marked on one side than on the other,-all these, as has been pointed out by Dr. Hughlings Jackson, closely resemble the attitude seen in ordinary hemiplegia due to a cerebral lesion.

Another symptom, which is seen only in advanced cases accompanied by much rigidity, consists in a peculiar method of assuming the recumbent attitude. The patient, instead of lying down in bed in the usual way, climbs up on to the bed and stands on it. Then bending down very slowly he takes hold of the rail at the foot of the bed with both his hands and gradually sits down on the bed, holding on to the rail all the time. Having in this manner reached the sitting posture the patient then allows his trunk to fall backwards into the recumbent posture. This circuitous method of getting into bed is characteristic of the disease. A somewhat similar affection is described below (vide Case 3). Difficulty in changing the position when in bed is a common symptom and patients frequently complain, in addition, of a difficulty in finding a comfortable position when lying down. This feeling of restlessness is interesting when we remember the muscular rigidity which accompanies it.

In concluding this paper I should like to call attention to a new symptom which has not hitherto, I believe, been placed on record. As might be expected, it is a minor symptom, but in my series of cases it occurred no fewer than five times-i.e., with sufficient frequency to place it beyond 
the category of mere coincidence. It consists in a peculiar affection of the toes, which in the majority of the cases in which I have met with it was one of the earliest symptoms of the disease, and is therefore, I think, of considerable diagnostic value. It is as follows. The patient complains, when walking, that the toes of one foot occasionally become spontaneously strongly flexed and curled up under the sole in a cramp-like fashion, causing difficulty in walking. This "curling up" of the toes is often so uncomfortable that the patient has to stand still for a minute or two until he can get his toes to relax and spread out flat again. All the toes, with the exception of the great toe, participate in this flexor contracture; the hallux, on the other hand, usually becomes hyperexlended at the same time; sometimes, however, it may become flexed like the other toes. In some cases the contraction may spread to the anterior tibial muscles, causing an inversion of the ankle as well. For permission to publish my notes $I$ am indebted to the courtesy of Dr. Hughlings Jackson, Dr. Buzzard, Dr. Ferrier, and Dr. Ormerod.

CASE 1.-The patient, a man, fifty-four years of age, was admitted to the hospital on Dec. 13th, 1896. Six months before his admission, when walking, he began to feel the toes of both feet become occasionally hyperextended, this movement passing on at once to one of plantar flexion. His ankles also became dorsiflexed, so that he had to take a step or two backwards until the cramp passed off. Two months after the onset of this symptom his hands became stiff and weak and his handwriting became abnormally slow. On admission he had the typical facies of paralysis agitansthe starched expression, the stooping of the head, the elbows slightly flexed, the fingers in the "interosseal" position, no tremor either at rest or on movement, and all voluntary movements slow and stiff.

CASE 2.-A man, forty-two years of age, was admitted to the hospital on Feb. 9th, 1897. For four years before his admission he noticed that the toes of his right foot would occasionally become strongly flexed when he walked so that he had to stand still until his toes relaxed. Later his right leg became stiff and weak and his right hand became slow in its movements so that he could not cut his food readily nor could he write so easily or so rapidly as usual. Within the last three or four months he had developed slight tremor of the right foot when fatigued. On admission he had slight weakness and rigidity of the right face, arm, and leg, with marked "starched" expression. The right hand was held in the "interosseal" attitude and the right elbow was habitually semi-flexed. All movements of the right arm and leg were slower and stiffer than on the left side. He dragged his right foot a little in walking; there were no tremors on admission. He complained of difficulty in turning in bed and in finding a comfortable posture when in bed.

CASE 3.-A married woman, aged fifty years, was admitted to the hospital on June 14th, 1898. For three years she had had trembling of the left hand and arm followed, some six months later, by trembling of the right hand, which she first noticed when putting pegs on a clothes-line. For two years she had had difficulty in turning in bed and had been unable to get into or out of bed without assistance owing to stiffness of her legs. About a year before admission she began to feel her toes curling up under her soles inside her shoes when she was sitting down. This made her get up and walk about for a few minutes until the symptom passed off. She had been unable to sing for a year; within the last month or so her jaw began to tremble and her articulation became less distinct. On admission she was seen to have severe paralysis agitans with the typical "starched" face, the typical attitude of the limbs and trunk, and tremors of the face, jaw, tongue, and limbs, synchronous, the rate of tremor being 6 per second. The tremors affected the limbs equally on the two sides. Her articulation was markedly festinant and her voice was monotonous and plaintive. All voluntary movements of the trunk and limbs were slow and stiff and the tremor persisted in spite of voluntary movement. The gait was slow and shuffing and there was no festination; she could not turn round without holding on to the furniture. She could not feed herself owing to tremors. She was unable to lie down in bed herself. When trying to do so she put one knee on the edge of the bed and then fell heavily on to the bed in a semi-prone posture, from which she was unable to move without assistance. She perspired excessively and would not allow herself to be covered by more than a single sheet.

CASE 4.-A married woman, aged fifty jears, was admitted to the hospital on March 1st, 1897. Her mother's sister had paralysis agitans. Four years previously, a fortnight after her last confinement, the patient noticed tremor of the fingers of the left hand on movement. Stiffness of the left arm and later of the left leg had since developed. Within the last two years the right side had become stiff, the arm being affected before the leg. For about two years she had noticed in walking that the toes of both feet occasionally curled under the soles, except the big toes, and she had to stand still for a few minutes until this passed off. She had had great difficulty in turning in bed for the past six months. On admission she had the typical face of paralysis agitans, "interosseal" attitude of the hands, stooping head, festinant shufling gait, and marked retropulsion. The speech was monotonous and festinant. All the voluntary movements were slow and stiff, especially on the left side. She had difficulty in doing her hair herself; she could not use her left hand easily to "gather" the hair There was very slight tremor in the left hand, this occurring only on holding it out, not when it was at rest.

CASE 5.-A man, fifty-three years of age, was admitted to the hospital on Dec. 4th, 1896. He was left-handed. For four years he had noticed that the toes of his right foot occasionally became stiffy flexed into the sole; the hallux, however, became hyperextended. About two years previous to his admission he began to bave slowness and tremor in writing with the right hand, and about a year afterwards his right leg began to shake, especially in walking. On admission he had the typical symptoms of paralysis agitans in the right face, arm, and leg, there being both tremors and rigidity. The gait was shuffling but not festinant. He could voluntarily stop the tremors for a moment.

In 3 of the above 5 cases the "toe-curling" symptom was the earliest to appear, preceding the other symptoms by intervals of four months (Case 1), a year (Case 2), and two years (Case 5) respectively.

Since the above paper was written I have been kindly favoured by Dr. Ormerod (to whom I had mentioned this symptom) with the notes of another corroborative case in which the patient, after having had the "toe-curling" symptom for five years, developed symptoms of paralysis agitans.

CASE 6. - The patient, a man, aged forty-four years, consulted Dr. Ormerod in March, 1892, for a "drawing under of the toes of the left foot. This came on chiefly after walking, all the toes becoming flexed, beginning with the big toe and then the ankle becoming inverted. The symptom had been present since 1890 . Dr. Ormerod writes: "At that time I do not think there was anything in his symptoms or general appearance to make me suspect that he had paralysis agitans. In May, 1895, he came again and there was no doubt that he now had paralysis agitans. There were characteristic tremors of both hands and the facies was that of paralysis agitans; the gait was not very characteristic. The affection of the left foot still continues; it looks shorter and more humpy than the right."

Queen-square, W.C.

\section{Clinital aldets:}

\section{MEDICAL, SURGICAL, OBSTETRICAL, AND} THERAPEUTICAL.

\section{TWO CASES OF PUERPERIUM COMPLICATED WITH} SCARLET FEVER; RECOVERY.

By J. Gawler MurRaY, L.R.C.P., L.R.C.S. EdiN., L.F.P.S. GrASG.

THE following cases may be of interest, showing that pregnant women seldom develop scarlet fever (Haultain).

CASE 1.-I attended the patient on May 24th. The child was born on my arrival and the placenta came away quite easily. I visited the patient on the 26th, 27th, and 28th, her temperature never being above $99.2^{\circ} \mathrm{F}$. On the 29 th $I$ found her looking very ill and with a temperature of $105^{\circ}$. I immediately douched out the uterus with iodine and water and ordered the nurse to give a vaginal douche of weak Condy's fluid every two hours as the discharge was very foul-smelling. I called in the afternoon to repeat the intrauterine douche and found the patient's temperature to be 